\title{
A comparative study of platelet rich fibrin and bone graft in intrabony defect treatment: A systematic review
}

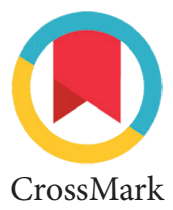

\author{
Arni I. Djais, Heri Siswanto*
}

\section{Abstract}

Objective: To compare the result of treatment using platelet rich fibrin and bone graft in intrabony defect cases.

Methods: Pubmed and Wiley online searches were conducted to identify articles published in dental journals from January 2013 to December 2017 focusing on platelet rich fibrin and bone graft in intrabony defect treatment. Manual searches of published full-text articles and related reviews were performed afterwards. There are 135 studies found, but only 4 studies met the inclusion criteria. Results: The initial database search produced 135 articles. All articles were selected for full-text review. A total 4 studies were selected for inclusion, with 107 patients as subjects. All of the studies showed the decreased of probing depth (PD), clinical attachment loss (CAL), intrabony defect (IBD) or radiograph defect depth (RDD) after 9 months follow up. The differences between baseline and clinical outcome measurement showed that single treatment using bone graft is the best result.

Conclusion: single treatment using bone graft showed better result than other treatments, such as PRF or combination PRF and bone graft.
Department of Periodontology, Faculty of Dentistry, Hasanuddin University, Makassar, Indonesia
*Correspondence to: Heri Siswanto, Department of Periodontology, Faculty of Dentistry, Hasanuddin University, Makassar, Indonesia herisiswant099@gmail.com

Received: 8 June 2018

Revised: 10 June 2018

Accepted: 9 July 2018

Available Online: 1 December 2020

Keywords: Alveolar bone loss, Bone grafting, Periodontal bone loss, Platelet rich fibrin

Cite this Article: Djais Al, Siswanto H. 2020. A comparative study of platelet rich fibrin and bone graft in intrabony defect treatment: A systematic review. Journal of Dentomaxillofacial Science 5(3): 137-140. D0I: 10.15562/jdmfs.v5i3.747

\section{Introduction}

Periodontitis is an inflammatory disease characterized by destruction of alveolar bone, root cementum, periodontal ligament and gingiva as a response to insults elicited by microbial accumulations on tooth surfaces. These responses tend to result in variety of intraosseous defects of various architectures. Periodontal therapy is performed with the primary objectives of gaining access to the diseased sites, achieving reduction in pocket depth, arresting further disease progression and finally restoring the periodontal tissues lost due to disease process. The ultimate aim is to achieve periodontal regeneration via new attachment formation. Regeneration has been defined as the reproduction or reconstitution of the lost or injured part to restore the architecture and function of the periodontium. ${ }^{1}$

Bone graft is the appropriate action to increase the height of alveolar lingir, jaw bone remodeling, microvascular free tissue transfer, and alveolar crest re-formation. Generally, bone graft is divided into four categories, namely autografts / autogenous, bone tissue derived from the same individual, allografts, bone tissue from different individuals, heterografts, bone tissue of different species (animals), alloplastic grafts, bone tissue using synthetic bone such as hydroxylapatite, phosphoric calcium ceramics, and oily calcium hydroxide in cream form. Therefore, bone allografts harvested from another human cadaver has been the most common alternative. Freeze or fresh-frozen bone, freeze-dried bone allograft (FDBA) and demineralized freeze-dried bone allograft (DFDBA) have all been utilized successfully for the regeneration of intrabony/furcation defects. Similarly, while certain countries do not allow the use of allografts, xenografts derived from various animal sources have also been widely used. Lastly, alloplasts are synthetically developed bone replacement grafts fabricated from various laboratory materials including hydroxyapatite and betatricalcium phosphate. ${ }^{2,3}$

In recent years, researchers have focused on biological mediators which have the ability to enhance wound healing and improve clinical benefits of bone replacement grafts.6 Polypeptide growth factors are biological mediators which regulate cell proliferation, chemotaxis and differentiation and induce periodontal regeneration. To deliver high concentrations of polypeptide growth factors to periodontal surgical wounds, use of autologous platelet concentrates serve as a safe and appropriate approach. Various platelet concentrates have been developed to improve soft and hard tissue healing. Platelet-rich fibrin (PRF) is a second-generation platelet concentrate, defined as autologous leukocyte- and platelet-rich fibrin because it collects 
leukocytes and platelets with high efficiency. PRF has been demonstrated to release polypeptide growth factors such as transforming growth factor $\beta 1$, platelet-derived growth factor, vascular endothelial growth factor and matrix glycoproteins (such as thrombospondin-1) gradually over at least 1 week. ${ }^{4-6}$

The aim of the present review is to systematically evaluate the effect of treatment using platelet rich fibrin and/or bone graft in intrabony defect cases by obseving clinical or radiological outcomes.

\section{Methods}

This systematic review was written according to the guidelines of PRISMA (Preferred Reporting Items for Systematic reviews and Meta-Analyses) for reporting studies evaluating healthcare interventions. PICO question (population, intervention, control, outcome) of the present systematic review was: P: patient with intrbony defects, I: Open flap operation, C: treated with PRF or PRF+Bone graft, $\mathrm{O}$ : Campairing the clinical result and radiograph between treatment with PRF or PRF+Bone graft.

Initial Pubmed and Wiley search of the English language literature was performed to establish a study protocol. These searches were conducted to identify articles published in dental journals from January 2013 to December 2017 focusing on study of Platelet Rich Fibrin and Bone Graft in Intrabony Defect Treatment. The MeSH (NCBI PUBMED) keywords used were "alveolar bone loss" and "bone grafting" and "platelet rich fibrin". The search limits applied to the electronic search were the Article types, search period. Manual searches of published full-text articles and related reviews were performed afterwards. There are 40 studies have shown on this matter in Pubmed, and 95 studies in Wiley, with only 3 studies met the inclusion criteria. Specific keywords were used to identify the appropriate studies needs, and followed the characteristics of PICO question.

The inclusion criteria in this systematic review were the following: english language article, full text article, any clinical study published between January 2013 to December 2017, that the studies reported on treatment intrabony defects with PRF or PRF+bone graft, that the studies included information about Platelet Rich Fibrin or/and Bone graft, that the studies included a clinical evaluation of the outcome with mean follow-up of at least nine months after treatment.

The exclusion criteria were all studies which did not satisfy the above mentioned criteria, such as animal studies and systematic review and meta analysis.
Specific keywords were used by two participating authors (HS and $\mathrm{AI}$ ) resulted the selection of the papers based on reading of abstract and fulltexts. Independently, the two investigators selected the paper based on inclusion criteria formerly set. After that, all abstracts and full-texts were downloaded and individually evaluated. The eligibility criteria were used to identify the articles that will be used for this systematic review.

The data were retrieved by two reviewers (HS and AI) that regarding following parameters: authors; year of publication; number of patients; technique; and objective. All of the full-texts which met the inclusion criteria were read independently by two reviewers, and evaluated to formulate this systematic review.

\section{Results}

The database search yielded 135 references, including 40 from PubMed and 95 from Wiley. After removing duplicates references, there were 11 studies remained. The titles and abstracts were reviewed afterward, and 4 studies were eligible for further analysis. The full-texts then be reviewed by the investigators and yielded 4 articles which met the inclusion criteria. The flowchart of article selection is shown in figure 1 with total 4 selected articles from initial yield of 135 studies by electronic literature search. After 135 titles reviewed, 11 articles were selected for this systematic review inclusions, whereas the other 7 articles were excluded for some different reasons.

The four studies were published between 2013 and 2017. The number of participants in the studies were 107 patients with 190 sites. All of the studies dealt with intrabony defects. Data from studies were combined in order to evaluate the treatment effect between platelet rich fibrin and platelet rich fibrin + Bone graft. Each study provided the mean clinical attachment level (CAL,); probing depth (PD), some studies use intrabony defects (IBD) or radiograph defect depth (RDD) measured at baseline and nine months follow up. The clinical parameters showed in table 1.

Patel et al. ${ }^{7}$ showed that Mean values after 9 months follow up of PD and CAL from PRF + OFD was $3.8 \pm 1.48$ and $3.60 \pm 0.7$ and OFD only was $1.9 \pm 1.2$ and $1.50 \pm 0.70$. Bajaj et al. ${ }^{8}$ also showed the 9 months follow up of PD and CAL and IBD from PRF + OFD was $3.14 \pm 1.26$ and $2.66 \pm 1.9$ and $2.24 \pm 0.66$ and OFD only was $2.14 \pm 1.26$ and $1.59 \pm 1.01$ and $0.84 \pm 0.99$.

Galav et al. ${ }^{9}$ showed that Mean values after 9 months follow up of PD and CAL and IRD from PRF only was $4.1 \pm 0.63$ and $3.9 \pm 0.57$ and $5.76 \pm$ 0.83 and $A B G$ only was $4.89 \pm 0.57$ and $4.50 \pm 0.61$ 
Table 1 Descriptive data from Clinical Parameters (mean \pm SD) of PD, CAL, IBD, RDD (mm), and between PRF and PRF+BG and BG

\begin{tabular}{|c|c|c|c|c|c|c|c|c|c|c|}
\hline \multirow{2}{*}{ Author } & \multirow{2}{*}{$\begin{array}{l}\text { Subject } \\
\text { (sites) }\end{array}$} & \multirow[b]{2}{*}{ Intervension } & \multicolumn{4}{|c|}{ Baseline } & \multicolumn{4}{|c|}{ Clinical Outcome } \\
\hline & & & PD & CAL & IBD & RDD & PD & CAL & IBD & RDD \\
\hline \multirow{2}{*}{ Patel et al } & \multirow{2}{*}{$13(26)$} & $\mathrm{PRF}+\mathrm{OFD}$ & $8.3 \pm 1.63$ & $7.1 \pm 0.73$ & & & $4.5 \pm 0.52$ & $3.5 \pm 0.52$ & & \\
\hline & & OFD & $7.90 \pm 1.19$ & $6.8 \pm 1.13$ & & & $6.0 \pm 0.81$ & $5.3 \pm 0.82$ & & \\
\hline \multirow{2}{*}{ Bajaj et al } & \multirow{2}{*}{$17(54)$} & $\mathrm{PRF}+\mathrm{OFD}$ & $(8.03 \pm 1.19$ & $6.77 \pm 0.84$ & $4.81 \pm 0.51$ & & $4.88 \pm 1.05$ & $4.11 \pm 0.75$ & $2.57 \pm 0.49$ & \\
\hline & & OFD & $7.96 \pm 1.28$ & $4.11 \pm 0.75$ & $4.71 \pm 0.71$ & & $5.81 \pm 1.07$ & $5.14 \pm 0.86$ & $3.86 \pm 0.54$ & \\
\hline \multirow{2}{*}{ Galav et al } & \multirow{2}{*}{$20(20)$} & PRF & $7.30 \pm 1.76$ & $12.60 \pm 1.43$ & & $5.76 \pm 0.83$ & $4.79 \pm 0.97$ & $8.70 \pm 1.05$ & & $4.55 \pm 0.70$ \\
\hline & & $\mathrm{ABG}$ & $7.40 \pm 1.57$ & $13.10 \pm 1.05$ & & $5.89 \pm 0.73$ & $4.82 \pm 1.39$ & $8.60 \pm 1.07$ & & $4.10 \pm 0.47$ \\
\hline \multirow{3}{*}{ Pradep et al } & \multirow{3}{*}{$57(90)$} & PRF & $8.17 \pm 1.26$ & $6.40 \pm 1.13$ & $5.63 \pm 1.16$ & & $4.27 \pm 0.69$ & $3.37 \pm 0.56$ & $2.43 \pm 0.68$ & \\
\hline & & $\mathrm{PRF}+\mathrm{HA}$ & $8.37 \pm 1.19$ & $6.65 \pm 1.10$ & $6.03 \pm 1.16$ & & $4.10 \pm 0.85$ & $2.97 \pm 0.93$ & $2.17 \pm 0.99$ & \\
\hline & & Control & $8.03 \pm 1.13$ & $6.57 \pm 1.17$ & $5.80 \pm 0.81$ & & $5.07 \pm 0.74$ & $3.90 \pm 0.76$ & $4.87 \pm 1.04$ & \\
\hline
\end{tabular}

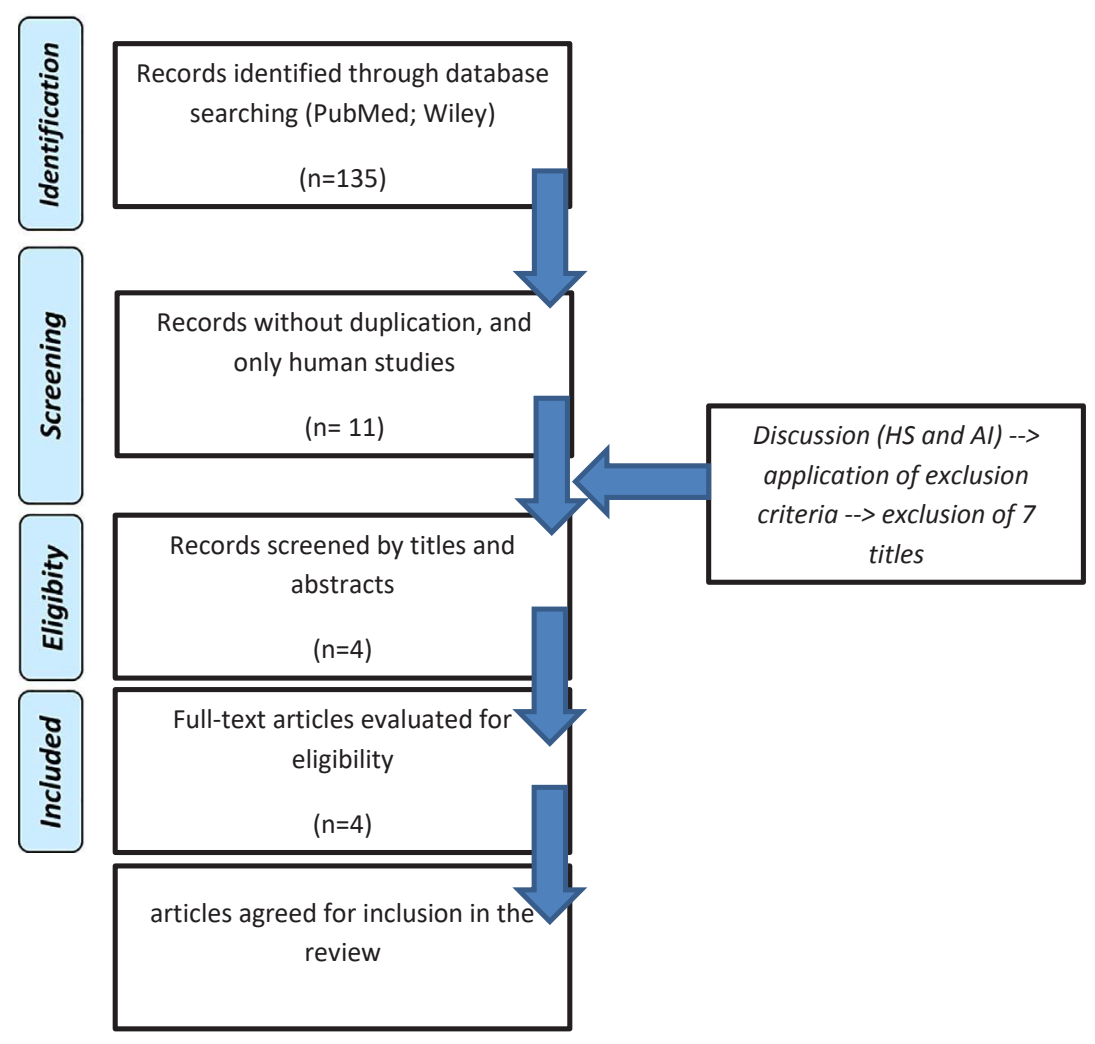

Figure 1 Article selection flow chart

and 5.89 \pm 0.73 . Pradeep et al. ${ }^{10}$ also showed the 9 months follow up of PD and CAL and IBD from $\mathrm{PRF}+\mathrm{OFD}$ was $3.9 \pm 1.09$ and $3.03 \pm 0.83$ and $3.20 \pm 0.89, \mathrm{PRF}+\mathrm{HA}+\mathrm{OFD}$ was $4.27 \pm 0.98$ and $3.67 \pm 1.03$ and $3.87 \pm 1.33$ and OFD only was $2.97 \pm 0.93$ and $2.69 \pm 1.09$ and $0.93 \pm 0.83$.

\section{Discussion}

Patel et al. ${ }^{7}$ reported that combination PRF and OFD has a better result than OFD only. The same result has been showed by Bajaj et al study. Meanwhile Pradeep et al reported that the combination of PRF and bone graft (Hydroxyapatit Bone graft) has a better result than combination of PRF and OFD or OFD only, even the results were better use bone graft and PRF in their study and it showed that the result were the best from the other studies. This may be caused by the type of bone graft they used in the study which is autologous bone graft. ${ }^{7-9}$

The ideal bone graft or bone graft substitute should provide three essential elements: an osteoconductive matrix; osteoinductive properties or factors; and osteogenic cells. Osteoconductivity can be defined as the process of infiltration of capillaries, perivascular tissue, and osteoprogenitor cells from the host bed into the transplant. The matrix need not be viable. However, as we will see, if the graft does not simulate the porosity and microstructure of human cancellous bone, incorporation into the bed will be delayed. Osteoinduction is the stimulation of a tissue to produce osteogenic elements. This process is controlled primarily by growth factors such as bone morphogenetic proteins (BMPs) that are capable of inducing differentiation of mesenchymal cells into cartilage and boneproducing cells. Osteogenetic cells are mesenchymal-type cells, and they can be summoned from host or graft bone marrow. The inclusion of osteogenic cells into grafts today remains procedurally difficult. Because few cells survive transplant, most osteogenic cells found in the graft are recruited there from the host bed by osteoinduction. This poses an obvious problem when the viability of the bed is compromised, such as a densely fibrotic defect. Thus, the creation of a bone graft or bone graft substitute that can function independently of the host bed condition is desirable. ${ }^{5}$

Amongst the material used in bone grafting, autogenous graft is considered the gold standard for reconstructions because it has properties such as osteoinduction, osteoconduction and osteogenesis. Despite that, a donor site is necessary increasing 
surgical morbidity as well as limited bone volume. For that reason, bone replacements are being developed and researched. Among these, anorganic bovine bone matrix is the most widely used both for its many years of research and its known osteoconductive potential. ${ }^{6}$

\section{Conclusion}

In this systematic review, we can conclude that intrabony defect treatment using bone graft (autologus bone graft) only showed a better result than other treatments, such as PRF or combination PRF and bone graft.

\section{Acknowledgment}

The author would like to express gratitude to Department of Periodontology Hasanuddin University, makassar, Indonesia, for their support to conduct the study.

\section{Conflict of Interest}

The authors report no conflict of interest.

\section{References}

1. Dr K. Malathi MD. Periodontal regeneration of an intrabony osseous defect with/ncombination of platelet rich fibrin and bovine derived $\backslash$ ndemineralized bone matrix: a case report. IOSR J Dent Med Sci 2013;4: 20-26.
2. Miron RJ, Mealey BL, Wang H. Use of Platelet Rich Fibrin for Periodontal Regeneration / Repair of Intrabony and Furcation Defects r r. 2017.

3. Drismayanti I, Jubhari EH. Perbandingan tingkat keberhasilan implan antara osteogenesis distraksi dengan autogenous bone graft Ratio of successful rate of dental implan between distraction osteogenesis with autogenous bone graft 2012;11: 180-186.

4. Sezgin Y, Uraz A, Taner IL, et al. Effects of platelet-rich fibrin on healing of intra-bony defects treated with anorganic bovine bone mineral. Braz Oral Res 2017;31: 1-11.

5. Sutherland D, Bostrom M. Grafts and bone graft substitutes. Bone Regen Repair 2005: 133-156.

6. Olate S, Duque-Netto H, Klüppel-LE, et al. Comparison of bone graft healing between autogenous bone, blood clot and anorganic bovine bone matrix: radiographic and histological analyses. Int J Morphol 2013;31: 1257-1262.

7. Patel KG, Gujjari KS, Kumar SCV. Platelet Rich Fibrin (PRF) in regeneration of intrabony defects- a randomized controlled trial. J Periodontol 2017;1: 1-14.

8. Bajaj P, Agarwal E, Rao NS, et al. Autologous platelet rich fibrin in the treatment of 3-wall intrabony defects in aggressive periodontitis - a randomized controlled clinical trial. J Periodontol 2017: 1-9.

9. Galav S, Chandrashekar K, Mishra R, et al. Comparative evaluation of platelet-rich fibrin and autogenous bone graft for the treatment of infrabony defects in chronic periodontitis: clinical, radiological, and surgical reentry. Indian J Dent Res 2016;27: 502 .

10. Pradeep AR, Bajaj P, Rao NS, et al. Platelet-Rich fibrin combined with a porous hydroxyapatite graft for the treatment of three-wall intrabony defects in chronic periodontitis: a randomized controlled clinical trial. J Periodontol 2012: 1-8.

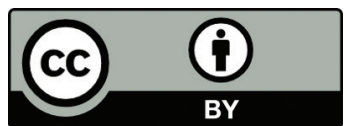

This work is licensed under a Creative Commons Attribution 